\title{
LESSON 17
}

MARGINS:

Left, Pica 20; Elite 30;

Right, moved out of the way.

Increase Speed

20 wpm 1 minute

S5 - SI 1.05

S6 - SI 1.10

Accuracy Practice

Improve Accuracy

15 wpm 1 minute

A $1-S I 1.06$

A2 - SI 1.26

A3 - SI 1.26

Test your Skill

1 minute

T1 - SI 1.25

The Clinic
It is usual to leave one line blank between paragraphs by turning up an extra line with the carriage return lever at the end of each. Do this every time you type your speed or accuracy work.

The three men tried to push the car to the brow of 10 the hill, but found it a very hot and tiring task. 20

The shop at the end of the road sells lots of good 10 food and they give you stamps with your groceries. 20

\begin{tabular}{|l|l|l|l|l|l|l|l|l|l|l|}
1 & 1 & 2 & 3 & 4 & 5 & 6 & 7 & 8 & 9 & 10
\end{tabular}

Just as you have learned that to improve your speed you must type easy passages within a given time and accuracy standard, you must also learn how to improve your typing accuracy. In speed drives, the emphasis is on speed-making your fingers move quickly - but in accuracy drives the emphasis is on accuracy first. In both speed and accuracy practice good typing technique is vital. All accuracy practices in this book are headed 'Improve Accuracy,' and before you type them you should read through the passages to try to spot the words you expect to slow you down because they are difficult. Practise these words until you can type them comfortably, and then practise them with the words before and after them. Once you have done this, try to type the passages in the time given with one error or fewer for each minute of timing. Some difficult words have been underlined: you may decide that these are not difficult for you and may practise others. The underlining is to remind you to type the difficult words first - you should not underscore them as you type each passage.

The fish would not bite all day so the boys packed 10 up and soon arrived home.

The snowman soon melted when the sun appeared, but 10 his hat and scarf remain.

There are many animals in the zoo, but I think all 10 creatures should be free.

A skill test is included in each unit of this book; you should see how much you can type in the time allowed. You may like to keep a record of your progress.

When you have typed this one minute test, you will 10 know how many words you can type in one minute and 20 how many errors you have made.

\begin{tabular}{|l|l|l|l|l|l|l|l|l|l|l|}
1 & 1 & 2 & 3 & 4 & 5 & 6 & 7 & 8 & 9 & 10
\end{tabular}

When you have typed a Test your Skill passage you should go through it and mark all your errors. Try to find out why you make mistakes and use the Clinic Index to find the drills required to correct your faults. Type the drills suggested. Remember the basis of all good typing is technique and concentration. The more time you spend erasing errors the slower will be your average typing speed, so take steps to cut errors to a minimum. 